\title{
VOLUNTAD PARA EL ESTUDIO Y EL TRABAJO ACADÉMICO EN ALUMNOS DE UNA UNIVERSIDAD PRIVADA DE LIMA
}

\author{
WILL FOR THE STUDY AND ACADEMIC WORK IN STUDENTS OF A PRIVATE UNIVERSITY OF LIMA
}

Héctor Hugo Sánchez-Carlessi', Elio Iván Rodríguez-Chávez', Patricia Roxana Matos-Ramírez’

\begin{abstract}
RESUMEN
Introducción: La voluntad para el estudio es una variable de interés de estudio por parte de toda institución educativa, ya que obedece a la política de mejorar la calidad del servicio educativo que brinda de forma constante. Objetivo: Identificar y comparar los niveles de voluntad para el estudio, considerando género y carrera de estudios en estudiantes de una universidad privada. Métodos: Estudio de tipo descriptivo comparativo, mediante la administración de la Escala de voluntad para el estudio, en una muestra de 762 estudiantes universitarios varones y mujeres de las carreras de Medicina humana, Psicología, Derecho, Ingeniería civil, Administración y gerencia, que cursan el IV, V y VI semestre de estudio. Resultados: Se encuentran diferencias significativas según género, en el sentido que las mujeres presentan mejores niveles de voluntad para el estudio en comparación con los varones. Se ha encontrado que en los alumnos de la carrera de Ingeniería hay menos voluntad para el estudio que en las otras carreras. Conclusión: Existen diferencias significativas en los niveles de voluntad para el estudio considerando género y tipo de carrera profesional de estudio.
\end{abstract}

Palabras clave: Voluntad para el estudio; Trabajo académico (fuente: DeCS BIREME).

\begin{abstract}
Introduction: Willingness to learn is an important study variable for any educational institution, since it is consistent with institutional policy of improving quality to the provided educational services. Objective: The main objective is to identify and compare the different levels of Willingness to Learn, considering the gender and different academic programs or careers, among the students of a private university. Methods: Descriptive and comparative study, through the administration of the Willingness to Learn Scale, in a sample of 762 male and female university students of Medical School, Psychology, Law, Civil Engineering, Administration and Management School (IVth, Vth and VIth academic semester). Results: We found significant differences considering gender; women show better or higher levels of Willingness to Learn, compared to men. Students of Engineering program showed a lower level of Willingness to Learn. Conclusion: We found significant differences among levels of Willingness to Learn, considering gender and academic program or career.
\end{abstract}

Key words: Willingness to learn; Academic work (source: MeSH NLM).

\section{INTRODUCCIÓN}

La voluntad para el estudio es un constructo psicológico que se ha convertido en un área de interés de investigación, ante la diversidad de estímulos distractores que pueden interferir en la vida académica de un estudiante universitario, como son los medios tecnológicos actuales, los cuales se encuentran al alcance de todos $s^{(1,2)}$.
La voluntad constituye una capacidad humana muy ligada a la conciencia y la motivación ${ }^{(3)}$. Es un estado de disposición de carácter cognitivoafectivo, relacionado con los intereses, los deseos, las motivaciones, la capacidad de toma de decisiones, y la autonomía en la regulación de la actuación de la persona para lograr algo ${ }^{(4,5)}$.

\footnotetext{
${ }^{1}$ Universidad Ricardo Palma, Lima-Perú.
}

Citar como: Héctor Hugo-Sánchez Carlessi, Elio Iván Rodríguez-Chávez, Patricia Roxana Matos-Ramírez. Voluntad para el estudio y el trabajo académico en alumnos de una universidad privada de Lima. Rev. Fac. Med. Hum. Abril 2020; 20(2):276-280. DOI 10.25176/RFMH.v20i2.2930 
Toda capacidad humana es un estado de disposición o potencial para realizar con éxito una actividad ${ }^{(6)}$. La voluntad como capacidad psicológica, está vinculada con el deseo, el interés y la motivación intrínseca y la fuerza del yo, que le permite a la persona ser persistente y tenaz para lograr un objetivo o meta ${ }^{(7-9)}$. Es la capacidad de decidir con libertad, y hacer una elección consciente sobre lo que la persona desea, quiere ser y hacer ${ }^{(10)}$.

La voluntad conforma una de las dimensiones básicas del comportamiento humano: corresponde a la dimensión volitiva, que compromete la conciencia humana, la racionalidad, el pensamiento, y rasgos afectivos ligados al carácter y la motivación ${ }^{(3,11)}$.

Son indicadores de la voluntad como capacidad humana: el deseo o interés por querer hacer algo que le atraiga, la actitud positiva para querer lograr algo, la fuerza del yo o fuerza de carácter, la toma de decisiones clara y definida, la motivación intrínseca y/o extrínseca y la tenacidad o perseverancia ${ }^{(12)}$.

En un estudio sobre el constructo psicológico de la voluntad ${ }^{(13)}$ en donde se elaboró una escala unidimensional de voluntad de trabajo, mediante un cuestionario de 21 items aplicado a 1141 estudiantes de segundo año de psicología de la Universidad de Buenos Aires, encontró que la voluntad de trabajo quedó caracterizada como la actitud por la cual una persona se aboca a sus obligaciones llevándola a cabo con responsabilidad, precisión, automotivación y sin dilaciones, aun cuando dichas obligaciones pudieran ser desagradables; asimismo, en la investigación titulada(14) "Delimitación del Constructo Voluntad de Trabajo", orientada a la construcción de una escala ad hoc. consideraron que "La voluntad de trabajo se caracterizó como la actitud por la cual un individuo se aboca a sus obligaciones con responsabilidad, eficiencia, automotivación y sin dilaciones".

El presente estudio tiene por objetivo efectuar un diagnóstico descriptivo de la voluntad para el estudio en estudiantes de una Universidad Privada de Lima considerando la carrera profesional y el género.

\section{MÉTODOS}

\section{Diseño del estudio}

Investigación descriptiva con diseño de carácter descriptivo-comparativo, para lo cual se ha aplicado una escala para evaluar la voluntad para el estudio y el trabajo académico a muestras de estudiantes de cuarto, quinto y sexto semestres académicos, de diferentes carreras profesionales, y de ambos géneros.

\section{Población y muestra}

La población objetivo la conformaron todos los estudiantes de la Universidad Ricardo Palma de Lima, que en el año 2019-I comprendió una población de 12,160 estudiantes.

La población real o accesible, ver Tabla 1, la conformaron 2412 estudiantes del IV, V y VI semestres académicos de las carreras profesionales de: arquitectura, ingeniería civil, administración y gerencia, traducción, medicina humana, psicología, biología, derecho.

\section{Procedimientos}

\section{La Escala de Voluntad para el estudio.}

Evalúa 6 indicadores (El deseo o interés y actitud por estudiar, la fuerza del yo o carácter firme, toma de decisiones clara y definida, motivación intrínseca y/o extrínseca, perseverancia y tenacidad y no postergación de acciones decididas), mediante 24 ítems o reactivos, cuya puntuación es de 1, 2 y 3 puntos de acuerdo con lo siguiente: $1=$ Nunca; 2 = A veces; y 3 = Siempre; el puntaje máximo a esperar fue de 72 y el mínimo de 24 .

El tiempo de aplicación, previo consentimiento informado de parte del alumno fue de 15 minutos como máximo.

\section{Análisis estadístico}

Para el procesamiento de la información se eligieron los siguientes estadísticos: Para la obtención de la Validez: La prueba KMO y Bartlett; Para la obtención de la confiabilidad: Alfa de Cronbach; Para la Curva de Normalidad: La prueba de Kolmogorov-Smirnov; Para la estadística descriptiva de los alumnos por Carrera profesional: Media aritmética, Mediana, Desviación Estándar, Varianza, Rango; Para la estadística inferencial comparando género, carrera profesional y semestres académicos: La prueba $\mathrm{t}$ de student y el análisis de varianza.

\section{RESULTADOS}

Del total de participantes en el estudio, encontramos que la media fue de 21,71 años, predominando el sexo femenino con $53,81 \%$ de la muestra. Las demás características se pueden observar en la tabla 2.

Al obtener los resultados de puntaje de la Escala de Voluntad para el estudio según sexo, hallamos que en los hombres la media fue de 56,02 y en el caso de las mujeres, la media fue de 58,45. La comparación del puntaje de acuerdo a los demás factores evaluados se encuentran en la tabla 3.

Los resultados de la prueba post hoc de Bonferroni muestra que los estudiantes que tenían de 26 a más años, tuvieron un mayor puntaje que los que tuvieron entre 20 y 25 años (Tabla 4).

El puntaje de la Escala de Voluntad para el estudio de acuerdo a carrera profesional y semestre académico se encuentra en la tabla 5. 
Tabla 1. Características Generales de los estudiantes evaluados según sexo, carrera y semestre.

\begin{tabular}{|c|c|c|c|c|}
\hline \multirow{2}{*}{ Carreras } & \multicolumn{3}{|c|}{ Semestre } & \multirow{2}{*}{ Total } \\
\hline & IV & $\mathbf{V}$ & VI & \\
\hline Arquitectura & 166 & 218 & 179 & 563 \\
\hline Ingeniería Civil & 230 & 182 & 163 & 575 \\
\hline Administración y Gerencia & 70 & 85 & 40 & 195 \\
\hline Traducción e Interpretación & 72 & 66 & 46 & 184 \\
\hline Medicina Humana & 174 & 153 & 205 & 532 \\
\hline Psicología & 66 & 66 & 47 & 179 \\
\hline Biología & 26 & 36 & 22 & 84 \\
\hline Derecho & 34 & 45 & 21 & 100 \\
\hline Total & & & & 2412 \\
\hline
\end{tabular}

Tabla 2. Características generales de los estudiantes evaluados según sexo, carrera y semestre.

\begin{tabular}{lcc} 
& Media & Desviación estándar \\
Edad & $\mathbf{2 1 , 7 1}$ & $\mathbf{3 , 9 9}$ \\
Sexo & Precuencia & \\
Masculino & 352 & $46,19 \%$ \\
Femenino & 410 & $53,81 \%$ \\
Carrera & & \\
Administración & 120 & $15,75 \%$ \\
Arquitectura & 125 & $16,40 \%$ \\
Biología & 60 & $7,87 \%$ \\
Derecho & 75 & $9,84 \%$ \\
Ingeniería & 65 & $8,53 \%$ \\
Medicina Humana & 150 & $19,69 \%$ \\
Psicología & 117 & $15,35 \%$ \\
Traducción & 50 & $6,56 \%$ \\
Semestre & & \\
Ciclo IV & 277 & $36,35 \%$ \\
Ciclo V & 292 & $38,32 \%$ \\
Ciclo VI & 193 & $25,33 \%$ \\
\hline
\end{tabular}

Tabla 3. Tabla que expresa la media y la desviación estándar del puntaje de la Escala de Voluntad para el estudio según sexo, semestre y edad.

\begin{tabular}{lcc} 
& Media & Puntaje \\
\hline Sexo & & p \\
Masculino & $56,02 \pm 8,19$ & \\
Femenino & $58,45 \pm 7,47$ & $<0,001^{*}$ \\
Semestre & & \\
IV & $56,41 \pm 8,32$ & \\
V & $57,80 \pm 7,87$ & $0,058 \dagger$ \\
VI & $57,90 \pm 7,22$ & \\
Edad (años) & & \\
De 17 a 19 & $57,78 \pm 7,93$ & \\
De 20 a 21 & $56,68 \pm 8,06$ & $0,023 \dagger$ \\
De 22 a 25 & $56,65 \pm 7,47$ & \\
De 26 a más & $59,25 \pm 8,04$ & \\
\hline
\end{tabular}

* Prueba t de Student. / + ANOVA.

Pág. 278 
Tabla 4. Prueba post hoc de Bonferroni del ANOVA de la escala de voluntad según edad.

\begin{tabular}{lccc} 
& 17 a 19 años & $\mathbf{2 0}$ a 21 años & $\mathbf{2 2}$ a $\mathbf{2 5}$ años \\
\hline 20 a 21 años & 0,826 & & \\
22 a 25 años & 0,802 & $>0,999$ & \\
26 años a más & 0,736 & 0,045 & 0,045 \\
\hline
\end{tabular}

Tabla 5. Tabla que describe la media y la desviación estándar del puntaje obtenido por cada carrera según ciclo cursado.

\begin{tabular}{|c|c|c|c|c|c|c|c|c|}
\hline \multirow[b]{2}{*}{ Carreras } & \multicolumn{2}{|c|}{ Ciclo IV } & \multicolumn{2}{|c|}{ Ciclo V } & \multicolumn{2}{|c|}{ Ciclo VI } & \multicolumn{2}{|c|}{$\begin{array}{l}\text { Consolidado de } \\
\text { los ciclos }\end{array}$} \\
\hline & Media & $\mathbf{p}^{*}$ & Media & $\mathbf{p}^{*}$ & Media & $\mathbf{p}^{*}$ & Media & $\mathbf{p}^{*}$ \\
\hline Administración & $56,48 \pm 7,91$ & & $58,78 \pm 7,76$ & & $58,16 \pm 7,72$ & & $57,66 \pm 7,81$ & \\
\hline Biología & $56,53 \pm 8,90$ & & $56,35 \pm 9,06$ & & $56,13 \pm 7,15$ & & $56,38 \pm 8,37$ & \\
\hline Arquitectura & $56,48 \pm 7,91$ & & $59,06 \pm 8,06$ & & $58,08 \pm 7,34$ & & $57,84 \pm 7,89$ & \\
\hline Ingeniería Civil & $52,13 \pm 9,61$ & 0,251 & $53,94 \pm 6,54$ & 0,044 & $58 \pm 7,27$ & 0,858 & $53,92 \pm 7,98$ & 0,0223 \\
\hline $\begin{array}{l}\text { Traducción e } \\
\text { Interpretación }\end{array}$ & $57,56 \pm 9,22$ & & $60,25 \pm 6,84$ & & $58,56 \pm 7,26$ & & $58,74 \pm 7,83$ & \\
\hline $\begin{array}{l}\text { Medicina } \\
\text { Humana }\end{array}$ & $57,68 \pm 6,73$ & & $56,65 \pm 8,29$ & & $58,22 \pm 7,71$ & & $57,50 \pm 7,59$ & \\
\hline Psicología & $55,33 \pm 8,60$ & & $59 \pm 7,99$ & & $58,67 \pm 6,84$ & & $58,02 \pm 7,94$ & \\
\hline Derecho & $57,96 \pm 9,36$ & & $57,52 \pm 7,02$ & & $55,46 \pm 5,96$ & & $57,27 \pm 7,69$ & \\
\hline
\end{tabular}

* ANOVA.

Los resultados de la prueba post hoc de Bonferroni del ANOVA del puntaje de la Escala de Voluntad para el estudio de acuerdo con la carrera profesional, muestra que todas las diferencias encontradas entre grupos corresponden al puntaje de la carrera de Ingeniería civil frente al resto de carreras. Las cuales corresponden a 0,$057 ; 0,032 ;>0,999 ; 0,341 ; 0,061 ; 0,022$ y 0,032 ; para las carreras de Administración, Arquitectura, Biología, Derecho, Medicina Humana, Psicología y Traducción e Interpretación respectivamente. El resto de las comparaciones entre grupos no mostraron diferencias estadísticamente significativas ( $p>0,999)$.

\section{DISCUSIÓN}

Con relación a los resultados considerando las variables género, edad, y carreras de estudio se ha encontrado que, en relación con el género, se ha evidenciado a través de las pruebas estadísticas realizadas que las estudiantes mujeres, obtienen mayores niveles de voluntad para el estudio que los varones. Estos hallazgos demuestran que las mujeres tienen más capacidad de poder manejar la mayoría de los aspectos inmersos en la práctica académica. Tal como lo evidencia un estudio realizado en 186 estudiantes de enfermería que a pesar de no encontrar diferencias significativa entre ambos sexos con respecto a las variables estudiadas resalta la capacidad de las mujeres en su manejo de las influencias externas durante sus estudios ${ }^{(15)}$. Sin embargo, Azañon et al. al evaluar a 979 universitarios españoles encontró que los varones tenían mayor capacidad en el control de situaciones, sentimientos y pensamientos negativos ${ }^{(16)}$.

Respecto a la edad, los resultados logrados han permitido encontrar diferencias entre los estudiantes que tienen menor edad y los que tienen mayor edad. En esa línea, Acevedo et al encontró en un grupo de estudiantes colombianos que la media de edad para abandonar los estudios universitarios fue de 22,1 años ${ }^{(17)}$. Además, los estudiantes de ingeniería de una universidad en Bogotá, Colombia demostraron tener menor capacidad de manejar situaciones de estrés o presión cuando son más jóvenes lo que desencadenar en algunos en el abandono de su carrera ${ }^{(18)}$.

Con relación a los semestres académicos, los resultados tampoco han demostrado algunas diferencias significativas sobre voluntad para el estudio entre los 
alumnos de IV, V y VI semestres académicos, mientras que según la carrera profesional de estudios se encontraron que los alumnos de ingeniería civil en el IV semestre presentan menos voluntad para el estudio que el resto de los alumnos de las diferentes carreras profesionales. Este problema entre los estudiantes de Ingeniería siempre ha representado una dificultad debido a la complejidad de las materias impartidas con relación a otras carreras ${ }^{(19)}$. Sin embargo, el uso de estrategias académicas y psicológicas puede representar una opción entre estos estudiantes. Esta afirmación fue comprobada por Rodríguez Pascual y Martínez Rosillo entre estudiantes de ingeniería a través de un curso de couching grupal que impacto efectivamente en el desarrollo académico de estos estudiantes así como en su desarrollo psicológico(20).

Por otro lado, los alumnos de Medicina Humana y Traducción e Interpretación presentan mayor voluntad para el estudio. En el $\mathrm{V}$ semestre, los alumnos de ingeniería civil presentan menor voluntad para el estudio en cambios los de Arquitectura, Psicología y Traducción e interpretación presentan mayor voluntad para el estudio. En el VI semestre académico, no se encuentran diferencias significativas entre los alumnos de las diferentes carreras profesionales.

\section{CONCLUSIÓN}

Concluimos que las estudiantes mujeres presentan mayores niveles de voluntad para el estudio que los varones en la población de estudiantes universitarios de Lima. Asimismo, los estudiantes de Ingeniería Civil presentaron menor puntaje en la escala de voluntad para el estudio en comparación con otras carreras.

Contribuciones de autoría: Los autores participaron en la génesis de la idea, diseño de proyecto, recolección e interpretación de datos, análisis de resultados y preparación del manuscrito del presente trabajo de investigación.

\section{Financiamiento: Autofinanciado.}

Conflicto de interés: Los autores declaran no tener conflicto de interés en la publicación de este artículo.

Recibido: 19 de febrero 2020

Aprobado: 30 de marzo de 2020

Correspondencia: Hector Hugo Sanchez Carlessi.

Dirección: Vicerrectorado de Investigación, Universidad Ricardo Palma, Avenida Benavides 5440, Santiago de Surco, Lima-Perú.

Teléfono: 999732421

Correo:hecsancar31@gmail.com

\section{REFERENCIAS BIBLIOGRÁFICAS}

1. Núñez JC. Aportaciones de la psicología educativa al proceso enseñanzaaprendizaje. 2008.

2. Cartagena Beteta M. Relación entre la autoeficacia y el rendimiento escolar y los hábitos de estudio en alumnos de secundaria. Red Iberoamericana de Investigación sobre Cambio y Eficacia Escolar (RINACE); 2008.

3. Turner JE, Husman J, Schallert DL. The importance of students' goals in their emotional experience of academic failure: Investigating the precursors and consequences of shame. Educational Psychologist. 2002;37(2):79-89.

4. Sánchez H, Delgado A. Principios, leyes y conceptos fundamentales de la psicología. Ed. Business Support Aneth

5. Tirado Segura F. Psicología educativa para afrontar los desafíos del siglo XXI. 2010

6. Sánchez H. Psicología de la Editorial Visión Universitaria

7. Bembenutty $H$, Karabenick SA. Academic Delay of Gratification in Conditionally-Admissible Minority College Students. ERIC; 1997.

8. Kuhl J. Volitional aspects of achievement motivation and learned helplessness: Toward a comprehensive theory of action control. En: Progress in experimental personality research. Elsevier; 1984. p. 99-171.

9. Wolters CA. Self-regulated learning and college students' regulation of motivation. Journal of educational psychology. 1998;90(2):224.

10. Perrenoud P. La construcción del éxito y del fracaso escolar: hacia un análisis del éxito, del fracaso y de las desigualdades como realidades construidas por el sistema escolar. Ediciones Morata; 1996.

11. Husman J, McCann E, Crowson HM. Volitional strategies and future time perspective: embracing the complexity of dynamic interactions. International Journal of Educational Research. 2000;33(7-8):777-799.

12. McCann EJ, Garcia T. Maintaining motivation and regulating emotion: Measuring individual differences in academic volitional strategies. Learning and individual differences. 1999;11(3):259-279.

13. Galibert MS, Abal FJP, Lozzia GS, Aguerri ME, Attorresi HF. Voluntad de trabajo en estudiantes de psicología. Anuario de investigaciones. 2007;14(1):269-275.

14. Abal FJP, Lozzia GS, Aguerri ME, Galibert MS, Attorresi HF. Evidencias acerca de la validez de constructo de la escala Voluntad de Trabajo. Anuario de investigaciones. 2008;15:283-289.

15. Montes-Hidalgo J, Tomás-Sábado J. Autoestima, resiliencia, locus de control y riesgo suicida en estudiantes de enfermería. Enfermería Clínica. 1 de mayo de 2016;26(3):188-93.

16. Garzón Azañón M, Barahona Esteban M. Diferencias personales en el perdón en universitarios españoles en función del sexo [Internet] [Tesis de Grado]. [España]: Universidad de Extremadura; 2018. Disponible en: http:// dehesa.unex.es/handle/10662/8538

17. Acevedo D, Torres JD, Tirado DF. Análisis de la Deserción Estudiantil en el Programa Ingeniería de Alimentos de la Universidad de Cartagena durante el Periodo Académico 2009 - 2013. Formación universitaria. 2015;8(1):35-42.

18. Arévalo $\mathrm{S}$, Lizeth $\mathrm{M}$. Modelo Representativo de Deserción Estudiantil Voluntaria en Carreras de Pregrado de la Facultad de Ingeniería de la Universidad Distrital Francisco José de Caldas [Internet] [Tesis de Grado] [Colombia]: Universidad Distrital Francisco José de Caldas; 2015 [citado 4 de abril de 2020]. Disponible en: http://repository.udistrital.edu.co/ handle/11349/2939

19. Caicedo RO, Atuesta SB, Caicedo JP. Análisis de la retención de estudiantes de ingeniería basado en la pérdida consecutiva de una misma asignatura. Un enfoque de Cadenas de Markov. Ingeniería Industrial Actualidad y Nuevas Tendencias. 2016;5(16):7-18

20. Rodríguez Pascual LP, Martínez Rosillo VM. Efectividad del coaching grupal sobre el desarrollo de la autorregulación del aprendizaje en estudiantes de ingeniería. Cuadernos de Investigación Educativa. junio de 2015;6(1):71-88. 